\title{
Personalentwicklung im Kontext der interkulturellen Kommunikation
}

\author{
Olga Rösch
}

\section{Zum Ursprung des Fachgebietes Interkulturelle Kommunikation}

Die Erweiterung der Europäischen Union in Richtung Osteuropa führt zu einer weiteren Zunahme von internationalen Wirtschaftsaktivitäten in deutschen Unternehmen. Dank den günstigen Rahmenbedingungen des gesamteuropäischen Marktes können auch mittlere und kleinere Unternehmen ihre Wirtschaftsaktivitäten auf dem osteuropäischen Markt nachhaltig ausbauen. Bei der Entwicklung von internationalen Unternehmenstätigkeiten spielt die Qualifizierung der Mitarbeiter für die Zusammenarbeit in den bi- bzw. multikulturellen Teams eine besonders wichtige Rolle. Die Bedeutung der interkulturellen Kompetenz für die Bewältigung von Arbeitsaufgaben in den interkulturellen Kontaktsituationen ist längst erkannt. Der Bedarf an Wissen und Können für Lösungen von kulturbedingten Kommunikationsstörungen im Geschäftsbereich verhalf dem Fachgebiet Interkulturelle Kommunikation zur weitreichenden Anerkennung.

Mit der Problematik der interkulturellen Kommunikation beschäftigen sich bekanntlich mehrere Wissenschaftsdisziplinen: Kulturanthropologie, Fremdsprachephilologien, kulturvergleichende Psychologie, Kommunikationswissenschaften (z. B. auch Ethnorhetorik), Wirtschaftsund Sozialwissenschaften, um hier nur einige zu nennen (vgl. Rösch, 1997). Das ist durchaus verständlich: Der Vorgang selbst, durch diverse fremdkulturelle Determination kollokiert, verursacht aufgrund seiner Komplexität allzu oft kommunikative Störungen und bietet auch deshalb viele interessante Aspekte für deren wissenschaftliche Untersuchung.

Die Problematik der Kommunikation mit kulturell Fremden ist in den USA seit den späten 50er Jahren ein aktuelles Forschungsthema. Die Beschäftigung mit derartigen Fragen stand und steht sicherlich im Zusammenhang mit den Erfordernissen der Innen- und Außenpolitik der USA als einer multikulturellen Gesellschaft und einer wirtschaftlich expandierenden Großmacht. Den Grundstein für das Fach Interkulturelle Kommunikation legte der bekannte amerikanische Kulturanthropologe Edward T. Hall mit dem 1959 erschienenen Buch „The Silent Language“. Die Genesis dieses Gründerwerkes und zugleich des Fachgebietes geht noch weiter zuruick auf ein Projekt der US-Regierung, die 1946 das Foreign Service Institut am State Department eingerichtet und bedeutende Linguisten, Psychologen und Kulturanthropologen für die Arbeit herangezogen hat. Das Ziel des Unterfangens bestand darin, neue Qualifizierungskonzepte für den diplomatischen und anderen auswärtigen Dienst auszuarbeiten und die amerikanischen Mitarbeiter für die Praxis der interkulturellen Kommunikation zu schulen. Das war für die „theoriegewohnten Geisteswissenschaftler" keine einfache Aufgabe, ihr Wissen für die praktischen Aufgabenlösungen anwenderorientiert zu didaktisieren. Aber eben aus der „Begegnung von Wissenschaft und Praxis“ entstand das neue Fachgebiet, für dessen Selbstverständnis die Praxisnähe und Interdisziplinarität charakteristisch geblieben sind (vgl. Roth, 2001:10).

Interkulturelle Kommunikation ist seit mehreren Jahrzehnten ein ausgesprochen internationales Forschungsthema mit unterschiedlichen Ausrichtungen. Als Beispiel sei hier nur eine in Deutschland weniger bekannte Forschungsrichtung, die russische Ethnopsycholinguistik genannt, die in der damaligen UdSSR in den 70er Jahren entwickelt wurde (siehe z. B. Leontjev/Sorokin/Tarasov, 1977). Im Rahmen des sogenannten Lakunen-Modells, das seinen Ursprung in der Problematik des Übersetzens von schriftlichen Texten hatte, ging es um eine systematische Erfassung, Operationalisierung und Klassifizierung von kulturgebundenen Kommunikationsunterschieden (zur Rezeption des Modells in Deutschland vgl. Schröder, 1999).

Anfang der 90er Jahre fand die Thematik der interkulturellen Kommunikation mit diversen Schwerpunkten auf unterschiedlichen Wissensgebieten der Forschung und Lehre Eingang in deutschen Universitäten. Besonders bekannt wurde im In- und Ausland die interkulturelle Germanistik, die für das Verstehen der deutschen Kultur und die Vermittlung des Deutschen als Fremdsprache neue methodisch-didaktische Impulse gab.

Neben dem rein wissenschaftlichen, „ideellen“ Interesse am Thema gab es also schon immer ein praktisches: Die Zunahme von Kontakten mit kulturellen Fremden in den internationalen Wirtschafts- und Wissenschaftskooperationen sowie in vielen anderen Kontexten im eigenen Lande führte zur Notwendigkeit, die kommunikativen und wirtschaftlichen Misserfolge mit ihren unterschiedlich weit reichenden negativen Folgen zu minimieren und die Verständigung nach Möglichkeit zu optimieren. Es entstand auch ein großer Bedarf an Schulungen (vor allem für Fachkräfte in der Wirtschaft und öffentlichen Verwaltung) und logischerweise an Schulungspersonal, das dieses Wissen vermitteln konnte. Die Hochschulen in Deutschland reagierten darauf mit neuen Studiengängen, in denen die Studierenden entsprechend qualifiziert wurden. ${ }^{1}$ Ein neuer Beruf entstand - interkul- 
tureller Trainer. So kam ein weiterer Aspekt des Fachgebietes hinzu - die Methodik der Vermittlung der fremdkulturellen Kompetenz.

Das Lehr- und Forschungsgebiet Interkulturelle Kommunikation baut also auf Erkenntnissen aus verschiedenen wissenschaftlichen Disziplinen. Zugleich widerspiegelt die hohe Interdisziplinarität des Faches objektiv die Gegebenheiten und Bedürfnisse der Realität. Seinen festen Platz unter den Hochschulfächern verdankt es sicherlich in erster Linie den Anforderungen des Arbeitsmarktes. Aber nicht nur Know-how-Vermittlung erfolgt in diesem Hochschulfach, sondern immer mehr wird erkannt, dass die Ausbildung der Studierenden insbesondere in den nicht geisteswissenschaftlichen Studienrichtungen (vor allen in den Ingenieurwissenschaften) auf dem Gebiet der Interkulturellen Kommunikation im hohen Maße persönlichkeitsbildend wirkt.

Durch einen übermäßigen Gebrauch des Begriffes Interkulturelle Kommunikation in der politischen Rhetorik (als ein positives, aktuelles Schlagwort) scheint ihm allerdings im Bewusstsein der Nichtfachleute etwas Inflationäres anzuhaften. Das hat jedoch die inhaltliche Entwicklung des Fachgebietes keineswegs gestört und führte eher zu einer schärferen Konturierung des Faches an den Hochschulen. Spezifizierungen lassen sich an diversen Bezeichnungen wie Interkulturelle Zusammenarbeit, Cross Culture, Diversity Management, Cultural Studies usw. erkennen. Als Oberbegriff für das umfassende Wissensgebiet bleibt Interkulturelle Kommunikation aber nach wie vor probat und der Sachlichkeit verpflichtet. Es geht nämlich immer wieder um den Erwerb von kulturellem Wissen und der kulturellen Kompetenz, die es einem erlauben, in einer interkulturellen Kontaktsituation kommunikative Zusammenhänge zu verstehen und angemessen zu handeln, in einem multinational besetzten Arbeitsteam arbeitsfähig zu bleiben und kulturell bedingte Konfliktsituationen zu meistern.

\section{Personalentwicklung als Wettbewerbsvorteil}

Die Globalisierung, die uns mit der EU-Osterweiterung vor unserer eigenen Haustuir erreicht hat, stellt uns vor neuen Situation und neuen Herausforderungen. Hier sei zunächst an eine bekannte Geschichte von zwei amerikanischen Autoren, R. R. Thomas und M. L. Woodruff², über eine vorgenommene Zusammenarbeit erinnert: „Die Giraffe, ein aufstrebender Handwerker im Holzgewerbe, hat soeben einen Preis erhalten. Sein Haus ist zum Giraffenhaus des Jahres ernannt worden. Nun bekommt die Giraffe Besuch von einem guten Freund und Fachkollegen, dem Elefanten. Die beiden planen, zukünftig enger zusammen zu arbeiten und der Elefant möchte sich das Haus ansehen. Das bekommt verständlicherweise beiden nicht sehr gut - dem Elefanten nicht und dem Haus auch nicht. Die Giraffe meint: 'Wenn wir wirklich hier zusammen arbeiten wollen, solltest du etwas abnehmen. Ich könnte zwar die Eingangtuir verbreitern, sie muss nun sowieso repariert werden, aber es ist schon ein bisschen eng mit dir drin. Was hältst du von einem Abonnement im Fitness-Studio?' Der Elefant hält das für eine akzeptable Idee, will aber erst die Räumlichkeiten im oberen Stockwerk besichtigen. Als sich der Staub gelegt hat, meint die Giraffe: 'Du solltest nach dem Fitnesskurs gleich auch Ballettstunden nehmen, damit du etwas leichter wirst. Sonst können wir in diesem Haus wohl kaum miteinander arbeiten.' Der Elefant hat inzwischen seine Wunden verbunden und sagt: 'Ich glaube kaum, dass Fitness-Studio und Ballettunterricht uns auf Dauer weiter bringen. Hast du mal daran gedacht, an deinem Haus etwas zu verändern?'“3

Wie gut haben wir uns denn selbst auf die Veränderungen in einem erweiterten Europa eingestellt? Wie nehmen wir hier in Deutschland die Reaktion unserer östlichen Partnern auf die Globalisierung wahr? Prozesse wie die künftige EU-Osterweiterung setzen positive Rahmenbedingungen für eine Kommunikation mit anderen Kulturen. Bieten derartige politische Änderungen nicht Anlass und Möglichkeiten zugleich, das eigene Konzept zur konfliktmindernden Begegnung mit anderen Kulturen zu uiberprüifen?

Die wachsende internationale Verflechtung wirtschaftlicher Aktivitäten ist die Realität von heute. Die Wirtschaft hat „das Haus“ tatsächlich verändert, indem sie ihre internationalen Unternehmungen, die immer interkulturell variablen Determinanten ausgesetzt sind, funktionsfähig machte. Dazu bieten erfolgreiche internationale Konzerne genügend Beispiele. Der grenzüberschreitende Austausch von Arbeit, Kapital, Wissen und Personal bleibt ein ständiger Lernprozess, den insbesondere die Großindustrie mit immer neuen, angepassten Konzepten, Strategien und Managementtechniken bewerkstelligt und weiter bewerkstelligen muss, wenn sie die Wettbewerbsfähigkeit nicht verlieren will. Erfahrungen und Wissen aus der Zusammenarbeit mit kulturell Anderen in multikulturellen Unternehmen fließen im Fachgebiet Interkulturelles Management zusammen, das ohne Grundlagen des Wissensgebietes Interkulturelle Kommunikation gar nicht bestehen kann.

Die Zukunftsfähigkeit eines Unternehmens hängt bekanntlich zu einem großen Teil von einer weitblickenden Personalentwicklungsplanung ab, die eine Antwort auf die Frage geben sollte, „mit welchen Mitarbeitern das Unternehmen seine Ziele in Zukunft realisieren will“ (Kuihn, 2001:3). In einem international agierenden Unternehmen sieht sie auf jeden Fall eine Qualifizierung der Mitarbeiter für die Zusammenarbeit mit und in fremdkulturell geprägten Arbeitsorganisationen vor. Dies geschieht bereits in den großen Unternehmen, wie z. B. der Siemens AG oder der Volkswagen AG. In Bezug auf die Art der Personalentwicklung handelt es sich hier meist um die berufsbegleitende Personalentwicklung, die sowohl eine Anpassungsqualifikation angesichts der neuen Kommunikationssituationen (z. B. Gemeinschaftsarbeit in einem multikulturellen Team) als auch eine positionsorientierte Befähigung (z. B. Auslandsentsendung und Übernahme einer größeren Verantwortung) bezweckt (Kühn, 2001:4). 
Die bekannte Definition des Begriffes Personalentwicklung beschreibt „die Summe aller personalbezogener Maßnahmen im Unternehmen [...], die dazu dienen, Mitarbeiter unter Berüicksichtigung ihrer individuellen und beruflichen Neigungen und Interessen in fachlicher und verhaltensmäßiger Hinsicht anforderungsgerecht zu qualifizieren“ (Kühn, 1985:6). In unserem Zusammenhang wird der Begriff Personalentwicklung im engeren Sinne gebraucht, d. h. es geht hier speziell um die Personalbildung bzw. berufliche Bildung, und zwar um die Fortbildung. Die Personalbildung stellt nach Olfert/Steinbuch (2001:419 f.) die Basisaufgabe der Personalentwicklung dar. Die erstere gewinnt aus mehreren Gründen zunehmend an Bedeutung. Diese sind:

- der wirtschaftliche und technologische Wandel, der immer neue Anforderungen an die Mitarbeiter stellt,

- die mit dem Wandel verbundene Notwendigkeit, immer mehr Entscheidungen in immer kürzerer Zeit zu treffen,

- das Erfordernis, auf allen Hierarchieebenen Selbstkoordination und Flexibilität der Mitarbeiter zu verbessern, um auf Veränderungen schnell und flexibel reagieren zu können,

- die Auseinandersetzung mit neuen Situationen setzt Innovationsbereitschaft voraus und verlangt Problembewusstsein und Kreativität,

- eine erhöhte Bereitschaft, die Konflikte auszutragen und Lösungen zu finden (vgl. Olfert/Steinbuch, 2001: $421 \mathrm{f}$.).

Die Personalbildung hat zum Ziel, eine angemessene Handlungskompetenz des Mitarbeiters zu entwickeln. Die üblichen sozialwissenschaftlichen Modelle teilen die Handlungskompetenz in drei Teilbereiche ein: in die soziale, fachliche und methodische Teilkompetenzen ein (vgl. Olfert/Steinbuch, 2001:422). In anderen Darstellungen wird von vier Teilbereichen der Handlungskompetenz ausgegangen: individuelle, soziale, fachliche und strategische (= methodische) Kompetenz. Die Handlungskompetenz in interkulturellen Zusammenhängen wird dann um die Fremdsprachenkenntnisse, um das kulturelle (Fakten)-Wissen, um das Wissen um die Besonderheiten des interkulturellen Handelns und die Fähigkeit, dies umsetzen zu können, erweitert. Die Umsetzung erfolgt mit Hilfe von Werkzeugen wie Empathie, Rollendistanz und Metakommunikationsfähigkeit u. a., die z. T. auch in anderen Kompetenzbereichen zu finden sind (vgl. Bolten, 2001). Bolten schlägt deshalb vor, die interkulturelle Kompetenz als Fähigkeit zu verstehen, ,individuelle, soziale, fachliche und strategische Teilkompetenzen in ihrer bestmöglichen Verknüpfung auf interkulturelle Handlungskontexte beziehen zu können. Unterschiedlich gegenüber intrakulturellen Handlungszusammenhängen ist dementsprechend die Qualität der einzelnen Teilkompetenzen.“ (vgl. Bolten, 2001:87 f.). Diese müssen für die Gegebenheiten der interkulturellen Kontaktsituation gezielt ausgebaut und trainiert werden.

Aber welche Eigenschaften und Merkmale von Kandidaten für Auslandspositionen werden als Grundvoraussetzung für die Auslandsentsendung berïcksichtigt, bevor man eine Fachkraft überhaupt einem interkulturellen Training unterzieht? Welche Anforderungen an die Auslandsmitarbeiter, an die sog. Expatriates, werden denn gestellt? Es gibt mehrere Erhebungen, die eine Antwort auf diese Frage geben (vgl. Bergemann/Sourisseaux, 2003:200 f.). Sie variieren zwar in Einzelheiten, stimmen aber im Kern weitgehend überein. Ohne auf die methodische Problematik bei der Erfassung dieser Kriterien einzugehen, sei hier als Beispiel das Ergebnis einer Studie zu Anforderungen an Expatriates von Wirth (1992:157) angefuihrt:

Genannte Anforderungen

Häufigkeit der Nennungen in \%

Fachliche Eignung:

- Fachliche Qualifikation 95

- Berufserfahrung

Persönliche Eignung:

- Persönlichkeitsbild (Selbstdisziplin, Ausgeglichenheit, Toleranz) 75

- Physische und psychische Belastbarkeit

Kulturbezogene Eignung:

- Sprachkenntnisse

- Vertrautheit mit der Unternehmenskultur

64

- Kommunikationsfähigkeit

- Lern- und Anpassungsfähigkeit

- Organisations- und Improvisationstalent

- Pädagogisches Geschick

Stabile Familienverhältnisse

(Mehrfachnennungen waren möglich)

Es gibt mehrere Instrumente interkultureller Personalentwicklung, mit denen in den Unternehmen gearbeitet wird, z. B. Coaching, Selbststudium, Informationsund Präsentationsseminare, Gruppendiskussion (international), Teilnahme an Bildungsangeboten der Hochschulen, Abordnung zur ausländischen Tochtergesellschaft, Urlaubsvertretung im Ausland, Mitnahme auf Auslandsreisen und vor allem interkulturelle Trainings. Bevorzugt wurden traditionell das Coaching und die Abordnung zur ausländischen Tochtergesellschaft. Erst seit weniger Jahren werden auch Ausbildungs- und Trainingsprogramme im größeren Umfang praktiziert, was auf die Einsicht in die Nuitzlichkeit dieser Weiterbildungsmaßnahme auch in der Personalentwicklung hindeutet (vgl. Fritz/Möllenberg, 2003:301 f.). Den Unternehmen steht inzwischen eine Reihe von ausgearbeiteten Trainingskonzepten zur Verfügung, um im Rahmen der berufsbegleitenden Personalbildung je nach Lernziel die interkulturelle Kompetenz der Mitarbeiter zu entwickeln. Hier als Beispiel vier Trainingsansätze im Vergleich, die je nach Intention und Dimension der interkulturellen Kompetenz eher kognitiv (vor allem 1 und 3), affektiv oder behavioral (zu unterschiedlichen Graden 2 und 4) orientiert sind. Sie entwickeln zunächst 


\begin{tabular}{|c|c|c|c|c|}
\hline Typ & 1. Kulturinformationsseminare & 2. Culture-Awarness (Sensibilisierung) & 3. Cultur-Assimilator & 4. Contrast-Culture-Training \\
\hline Ziele & $\begin{array}{l}\text { Faktenwissen über fremde } \\
\text { Kultur, ergänzt durch } \\
\text { Verhaltenstraining }\end{array}$ & $\begin{array}{l}\text { Bewusstsein der eigenen kulturellen } \\
\text { Prägung schaffen, Vorurteile } \\
\text { aufarbeiten }\end{array}$ & $\begin{array}{l}\text { Wissensvermittlung über } \\
\text { fremde Kultur und Kultur- } \\
\text { standards (Verhaltens- } \\
\text { muster, Werte und } \\
\text { Normen), Verhaltenstipps }\end{array}$ & $\begin{array}{l}\text { Eigene Kulturstandards und } \\
\text { die der Zielkultur aufzeigen, } \\
\text { Konflikte im Kontakt } \\
\text { verdeutlichen, schwierige } \\
\text { Situationen ausprobieren }\end{array}$ \\
\hline Methoden & $\begin{array}{l}\text { Referate, Diavorträge, Filme, } \\
\text { Lehrgespräche, Anekdoten }\end{array}$ & $\begin{array}{l}\text { Gruppendynamische und Sensitivity- } \\
\text { Methoden, z.B. Übungen mit gegen- } \\
\text { sätzlichen Kulturen }\end{array}$ & $\begin{array}{l}\text { Programmiertes Selbst- } \\
\text { lernen: Critical incidents } \\
\text { sind mit Multiple-Choice- } \\
\text { Antworten zu bearbeiten } \\
\text { und werden kommentiert }\end{array}$ & $\begin{array}{l}\text { Fallstudienarbeit, Rollen- } \\
\text { spiele mit Trainer aus } \\
\text { Zielregion }\end{array}$ \\
\hline $\begin{array}{l}\text { Zentrale } \\
\text { Themen }\end{array}$ & $\begin{array}{l}\text { Religion, Kulturgeschichte, } \\
\text { Tipps und Tabus, persönliche } \\
\text { Erfahrungen der Referenten }\end{array}$ & $\begin{array}{l}\text { Ängste und Hoffnungen bezüglich } \\
\text { Fremdheit, Stereotype und } \\
\text { Vorurteile }\end{array}$ & $\begin{array}{l}\text { Arbeits- oder aufenthalts- } \\
\text { relevante Kulturstandards } \\
\text { und Konfliktfelder }\end{array}$ & $\begin{array}{l}\text { Arbeits- oder aufenthalts- } \\
\text { relevante Kulturstandards } \\
\text { und ihr Hintergrund }\end{array}$ \\
\hline Stärken & Geringer Zeitbedarf & $\begin{array}{l}\text { Intensive Selbsterfahrung, allge- } \\
\text { meine Erkenntnisse, die weltweit } \\
\text { hilfreich sind, geringer Aufwand } \\
\text { und Kosten }\end{array}$ & $\begin{array}{l}\text { Flexibel planbare Einzel- } \\
\text { arbeit mit schriftlichen } \\
\text { Unterlagen oder CD-ROM }\end{array}$ & $\begin{array}{l}\text { Landes- bzw. regionen- } \\
\text { spezifisch, Praxisnähe und } \\
\text { Alltagsrelevanz }\end{array}$ \\
\hline Schwächen & $\begin{array}{l}\text { Informationen zum Teil sehr } \\
\text { subjektiv, kaum verhaltens- } \\
\text { relevant }\end{array}$ & $\begin{array}{l}\text { Wenig Bezug zur konkreten } \\
\text { Situation in einer bestimmten } \\
\text { Kultur, daher oft geringe Akzeptanz } \\
\text { durch Teilnehmer }\end{array}$ & $\begin{array}{l}\text { Hohe Selbstlernkompetenz } \\
\text { erforderlich, trockene Ver- } \\
\text { mittlung eines lebendigen } \\
\text { Lernstoffs, kein Verhaltens- } \\
\text { training }\end{array}$ & $\begin{array}{l}\text { Kaum geeignet, wenn Ziel- } \\
\text { kultur unbekannt oder } \\
\text { mehrere Zielkulturen }\end{array}$ \\
\hline
\end{tabular}

persönliche Eigenschaften des Mitarbeiters und schaffen günstigere Voraussetzungen für dessen Auslandseinsatz. Ein integrierendes und konsequent interdisziplinäres Trainings- und Ausbildungsmodell, das mehrere Erfolgskriterien berücksichtigt, ist ein Gegenstand aktueller methodischer Diskussionen in der Forschung (vgl. Fritz/ Möllenberg, 2003:303-305).

\section{Zur Realität der Personalbildung auf dem Sektor Interkulturelle Kommunikation}

Die Qualifizierung des Personals gehört zwar in das Konzept einer modernen Personalentwicklung. Aber es wird immer wieder festgestellt, dass die Vorbereitung der Mitarbeiter auf Auslandseinsätze unzureichend ist (vgl. Fritz/ Möllenberg, 2003; Frank, 2003). Besonders die kleineren und mittleren Unternehmen stehen „nicht ohne Skepsis“ (Kühn, 2001:3) den Personalentwicklungsmaßnahmen gegenuiber mit der Begründung, man sei dem Aufwand personell nicht gewachsen und finanziell uiberfordert. Insbesondere betrifft es die interkulturellen Trainingsmaßnahmen, deren Nutzen die KMU unterschätzen in der irrigen Annahme, die Weltoffenheit der Mitarbeiter genüge (vgl. z. B. Stoessel, 2002:84). Dabei verkennt man, dass es bei der interkulturellen Kompetenz um eine Schlüsselqualifikation der Mitarbeiter im Auslandsgeschäft geht! Wenn eine Fach- und/oder Führungskraft sich bereits im Auslandseinsatz befindet, so steht sie in der Regel unter Leistungs- und Zeitdruck, so dass es ihr kaum noch möglich ist, den kulturell bedingten Missverständnissen bzw. Kommunikationsstörungen auf den Grund zu gehen und durch metakommunikative Bearbeitung der Konfliktsituation eine für beide Seiten annehm- bare Lösung herbeizuführen. Es entstehen Reibungsverluste, Frustration, Resignation und finanzielle Einbußen. Eine gewisse Widersprüchlichkeit zwischen den steigenden Auslandsaktivitäten der Unternehmen auf der einen und der gleichzeitigen (in Kauf genommenen) mangelnden Vorbereitung der Mitarbeiter auf den Auslandseinsatz auf der anderen Seite ist besonders bei den KMU zu beobachten. Dieses Missverhältnis konnte auch im Rahmen des Projektes „Stereotype Einstellungen und Kulturstandards in der aktuellen interkulturelle Kommunikation mit Partnern aus dem östlichen Europa. Eine empirisch-analytische und diskursanalytische Untersuchung bei Wirtschaftsunternehmen im Land Brandenburg“, das an der TFH Wildau in enger Kooperation mit den KMU der Region durchgefuihrt wird ${ }^{5}$, vielfach bestätigt werden.

Neben der geläufigen Vorstellung, die Weltoffenheit des Mitarbeiters ersetze die interkulturelle Kompetenz, existiert eine weitere weitverbreitete irrtuimliche Annahme, die Auslandserfahrung erhöhe automatisch die interkulturelle Kompetenz des Mitarbeiters. Eine Studie der TU Braunschweig6 geht dieser Frage nach und liefert ganz andere Belege aus der Praxis. So wurden in deutschen und US-amerikanischen Unternehmen Befragungen durchgefuihrt, um u. a. den Zusammenhang zwischen dem Auslandsaufenthalt der Mitarbeiter und den Ausprägungen von interkulturellen Kompetenzen, wie interkulturelle Sensibilität, soziale Kompetenz und situationale Anpassungsfähigkeit, zu ermitteln (vgl. Graf, 2003:19 f.). Es wurde festgestellt, dass sich die interkulturelle Sensibilität bei Mitarbeitern ohne Auslandserfahrung kaum von der der Mitarbeiter im Auslandseinsatz im Laufe der ersten sechs Monaten unterscheidet. Mehr noch: Bei amerikanischen Auslandsmitarbeitern sank sie nach ei- 
nem halben Jahr sogar erheblich. Die soziale Kompetenz der deutschen und amerikanischen Auslandsmitarbeiter hat während der ersten 6 Monate des Auslandseinsatzes ebenfalls abgenommen und unterschied sich nachteilig von der der befragten Mitarbeiter ohne Auslandserfahrung. Die Fähigkeit zu situationsangepasstem Verhalten hat sich bei den Vertretern beider Kulturen durch den Auslandsaufenthalt kaum geändert. Diese zu Beginn des Auslandseinsatzes abnehmenden drei Fähigkeiten, die einen wesentlichen Bestandteil der interkulturellen Kompetenz ausmachen, lassen sich möglicherweise als Folge des Kulturschocks ${ }^{7}$ erklären, der bekanntlich phasenweise verläuft. Die interkulturelle Kompetenz in allen ihren Ausprägungen steigt laut Braunschweiger Studie erst nach einem längeren Arbeitsaufenthalt im Ausland. Den Höhepunkt erreichen die interkulturellen Kompetenzwerte bei Auslandsmitarbeitern erst während des fünften Jahres des Auslandseinsatzes. Dem Bericht über die Studie der TU Braunschweig lässt sich allerdings nicht entnehmen, wie die Mitarbeiter für ihren Auslandseinsatz vorbereitet wurden.

\section{Schlussbemerkung}

Bei den hier besprochenen Möglichkeiten der Qualifizierung für die Arbeit mit dem kulturell Fremden im Rahmen der Personalbildung ging es um die interkulturellen Trainings und andere Mittel und Wege als Maßnahmen „on the job“. Diese sind unter den Gegebenheiten eines international tätigen Unternehmens in Bezug auf die Personalentwicklung auch zweckmäßig und erforderlich. Die interkulturellen Trainings sind dabei nur eine der Möglichkeiten. Die crashkursmäßig verlaufenden Veranstaltungen haben natuirlich nicht den Anspruch und auch kaum eine Chance, die Problemkomplexität zu erfassen, was dazu führt, dass akademisch gebildete Führungskräfte sich $\mathrm{z}$. T. unterfordert fuihlen. Zur Problematik der Evaluation von interkulturellen Trainings wird z. Z. viel geforscht (vgl. Thomas, A./Hagemann, K./ Stumpf, S., 2003:257 ff.).

Dagegen bietet die für den Erwerb der interkulturellen Kompetenz erforderliche Ausbildung im Rahmen des Studiums den angehenden Ingenieuren, Betriebswirten, Verwaltungsangestellten u. a. unvergleichbar bessere Möglichkeiten, das Wissen und eine Qualifikation auf dem Gebiet der interkulturellen Kommunikation zu erwerben. Die Dauer und die Intensität der Ausbildung, die Interdisziplinarität und Methodenkombination, der Stoffumfang und die Obligation, sich mit der Materie auseinander zu setzen, lässt bei den jungen Menschen in ihrem produktivsten Lebensalter mit Sicherheit eine größere „Tiefenwirkung“ zurück. In das Hochschulfach Interkulturelle Kommunikation fließen Forschungsergebnisse aus diesem umfangreichen Wissensgebiet auf einem direkteren Wege ein und können nach einer entsprechenden methodisch-didaktischen Aufarbeitung als Lernstoff vermittelt werden. Das Hochschulfach Interkulturelle Kommunikation - unter welcher Bezeichnung auch immer - ist außerdem der Zielsetzung verpflichtet, zur allgemeinen und allseitigen Persönlichkeitsbildung beizutragen und nicht nur die handwerkliche Herangehensweise zu üben.

\section{Anmerkungen}

1 z. B. Ludwig-Maximilian-Universität München, TU Chemnitz, Friedrich-Schiller-Universität Jena u. a.

2 Thomas, R. R./Woodruff, M. L. (1999): Building a House for Diversity ...

3 deutsch zit. nach Hansen, K. (2002:10): Vielfalt leben ...

4 zit. nach Stoessel, A. (2002:84-85): Interkulturelle Kompetenz erweitern.

5 s. Rösch, O./Loew, R./Pfeifer, A.: Interkulturelle Kompetenz ...; in diesem Heft.

6 referiert nach Graf, A. (2003): Trugschluss Auslandserfahrung ...

7 Zum Begriff Kulturschock s. Wagner, W. (1999): Kulturschock Deutschland. ...

\section{Literaturverzeichnis}

Bergemann, Niels/Sourisseaux, Andreas L. J. (2003): Internationale Personalauswahl, in: Bergemann, N./Sourisseaux, A. L. J. (Hg.), Interkulturelles Management, S. 181-235, Springer-Verlag, Berlin/Heidelberg/u. a. 2003.

Bergemann, Niels/Sourisseaux, Andreas L. J. (Hg.) (2003): Interkulturelles Management, Springer-Verlag, Berlin/Heidelberg/New York 2003.

Bolten, Jürgen (2001): Interkulturelle Kompetenz, Erfurt 2001.

Bungarten, Theo (1999) (Hg.): Sprache und Kultur in der interkulturellen Marketingkommunikation, Beiträge zur Wirtschaftskommunikation Bd. 11, Veröffentlichungen des ARBUK, Attikon Verlag, Tostedt 1999.

Ertelt-Vieth, Astrid (1990): Kulturvergleichende Analyse von Verhalten, Sprache und Bedeutung im Moskauer Alltag. Beitrag zu einer empirisch, kontrastiv und semiotisch ausgerichteten Landeswissenschaft, Frankfurt a. M. 1990.

Frank, Sergey (2003): Der Lächerlichkeit preisgegeben, in: management \& training 3/2003, S. 12-15.

Fritz, Wolfgang/Möllenberg, Antje (2003): Interkulturelle Kompetenz als Gegenstand internationaler Personalentwicklung, in: Bergemann, N./Sourisseaux, A. L. J., Interkulturelles Management, S. 295-307, Springer-Verlag, Berlin/ Heidelberg/u. a. 2003.

Graf, Andrea (2003): Trugschluss Auslandserfahrung, in: managemnt \& training 3/2003, S. $19-21$.

Hansen, Katrin (2002): Vielfalt leben, in: Social Management 1/2002, S. $10-15$.

Kühn, Gundolf (1985): Personalentwicklung. Grundlagen und Methoden wirksamer und motivierender Mitarbeiterqualifikation zur Sicherung des Unternehmenserfolgs, in: RKW-Handbuch Führungstechnik und Organisation. HFO, 15. Lfg., 1.85 .

Leontjev, A. A./Sorokin, Ju. A./Tarasov E. F. (Hg.) (1977): Nacional'no-kul'turnaja specifika recevogo povedenija, (Nationalkulturelle Spezifik des Sprachverhaltens), Moskva, Isdatel'stwo Nauka 1977.

Olfert, Klaus/Steinbuch, Pitter A. (2001): Personalwirtschaft, Friedrich Kiehl Verlag GmbH, Ludwigshafen (9. Aufl.) 2001.

Rösch, Olga (1997): Interkulturelle Kommunikation als wissenschaftliches Lehr- und Forschungsgebiet, in: Wissenschaftliche Beiträge der Technischen Fachhochschule Wildau 1/1997, S. 112-115.

Rösch, Olga/Loew, Roswitha/ Pfeifer, Anke (2003): Interkulturelle Kompetenz - heute eine unerlässliche Schlüsselqualifikation. Zwischenbericht zu einem Forschungsprojekt an der TFH Wildau, in: Wissenschaftliche Beiträge der Technischen Fachhochschule Wildau 2003. 
Roth, Juliana (2001): Interkulturelle Kommunikation - ein neues Studienfach an deutschen Universitäten, in: Kulturknall, Themen Service für Presse, Hörfunk und Fernsehen, April 2001, S. 10-11.

Schröder, Hartmut (1999): 'Lakunen' und die latenten Probleme des fremdkulturellen Textverstehens - Anwendungsmöglichkeiten eines Modells der Ethnopsycholinguistik bei der Erforschung textueller Aspekte der internationalen Produktvermarktung, in: Bungarten, Theo (Hg.), Sprache und Kultur in der interkulturellen Marketingkommunikation“, Beiträge zur Wirtschaftskommunikation Bd. 11, Veröffentlichungen des ARBUK, Attikon Verlag, Tostedt 1999, S. 174-206.

Stoessel, Annette (2002): Interkulturelle Kompetenz erweitern, in: managerSeminare Heft 53/2002, S. 83-88.

Thomas, Alexander/Hagemann, Katja/Stumpf, Siegried (2003): Training interkultureller Kompetenz, in: Bergemann, N./ Sourisseaux, A. L. J. Interkulturelles Management, Springer-Verlag, Berlin/Heidelberg/u. a. 2003, S. 237-272.

Thomas, R. Roosvelt/Woodruff, Marjorie L. (1999): Building a House for Diversity. How a Fable About a Giraffe and an Elephant Offers new Strategies for Today's Workforce, New York et. al. 1999. In Deutsch erschienen 2001 im Gabler-Verlag unter dem Titel „Management of Diversity. Neue Personalstrategien für Unternehmen. Wie passen Giraffe und Elefant in ein Haus?"

Wagner, Wolf (1999): Kulturschock Deutschland. Der zweite Blick, Rotbuch-Verlag, Hamburg 1999.

Wirt, Ekkehard (1992): Mitarbeiter im Auslandseinsatz. Planung und Gestaltung, Gabler, Wiesbaden 1992.

\section{Autorin}

\section{Prof. Dr. Olga Rösch}

Technische Fachhochschule Wildau

Institut für Interkulturelle Kommunikation

Telefon +493375 508-367

E-Mail: roesch@sprz.tfh-wildau.de 